\title{
Diagnostic Value of Elastography, Strain Ratio, and Elasticity to B-Mode Ratio and Color Doppler Ultrasonography in Breast Lesions
}

This article was published in the following Dove Press journal: International Journal of General Medicine

\author{
Mahnaz Ranjkesh ${ }^{1,2}$ \\ Farid Hajibonabi (1D ${ }^{2-4}$ \\ Fatemeh Seifar (iD ${ }^{5}$ \\ Mohammad Kazem Tarzamni ${ }^{1,2}$ \\ Behzad Moradi ${ }^{1,2}$ \\ Zhila Khamnian $^{6}$ \\ 'Medical Radiation Sciences Research \\ Group, Tabriz University of Medical \\ Sciences, Tabriz, Iran; ${ }^{2}$ Radiology \\ Department, Tabriz University of Medical \\ Sciences, Tabriz, Iran; ${ }^{3}$ Student Research \\ Committee, Tabriz University of Medical \\ Sciences, Tabriz, Iran; ${ }^{4}$ Aging Research \\ Institute, Tabriz University of Medical \\ Sciences, Tabriz, Iran; ${ }^{5}$ Stem Cell \\ Research Center, Tabriz University of \\ Medical Sciences, Tabriz, Iran; ${ }^{6}$ Social \\ Determinant of Health Research Center, \\ Tabriz University of Medical Sciences, \\ Tabriz, Iran
}

Purpose: The aim of this study was to investigate the sensitivity, specificity, and diagnostic accuracy of sonoelastography (SE), strain ratio (SR), elasticity to B-mode (E/B) ratio, and color Doppler ultrasonography (US) in suspected breast lesions.

Materials and Methods: This prospective study was conducted on women referred to Alzahra university hospital of Tabriz for annual screening of breast cancer between May 2017 and December 2018. B-mode US, SE, and color Doppler imaging were conducted in females with suspected mammography reports. The lesions in B-mode were classified according to the Breast Imaging Reporting and Data System (B-RADS). The results of SE imaging were graded based on five-grade SE score. SR and E/B ratio of each lesion were also analyzed in SE images. Color Doppler findings were categorized from 0 (no visible vessel) to 2 ( $>$ two vessels) based on the vascularity of the tumor. Pathology results were used as the gold standard to measure the area under the receiver operating characteristic curve (AUC), sensitivity, specificity, and diagnostic accuracy of each modality.

Results: One-hundred and ten breast lesions of 104 women aged $42.05 \pm 10.33$ years were included in the study. Seventy-seven of the lesions were benign and 3 were malignant Sensitivity and specificity of $97.0 \%$ and $77.9 \%$ for B-mode US, $93.9 \%$ and $87.0 \%$ for SE score, $81.8 \%$ and $66.2 \%$ for color Doppler US, $72.7 \%$ and $77.6 \%$ for E/B ratio (cutoff: 1.05 ), and $77.3 \%$ and $79.6 \%$ for SR (cutoff: 1.90 ) were obtained, respectively. Addition of SE score to B-mode US increased the sensitivity to $93.9 \%$, specificity to $93.5 \%$, and AUC from 0.95 to 0.97. Cumulative color Doppler US with B-mode US did not enhance the diagnostic accuracy of B-mode US.

Conclusion: SE was more effective than color Doppler US for distinguishing malignant from benign breast lesion. Among the three different SE features, five-grade SE score was superior to $\mathrm{E} / \mathrm{B}$ ratio and $\mathrm{SR}$.

Keywords: breast cancer, elastography, color Doppler sonography, strain ratio, B-mode ultrasonography

\section{Introduction}

Breast cancer is the most common form of malignancy among females, worldwide. The high prevalence of disease and its progressive nature puts a great burden on health-care systems. ${ }^{1,2}$ Early diagnosis of malignant breast masses is essential for the effective management of malignancies. Mammography is the first-line imaging modality for breast cancer screening, which categorizes breast lesions according to standardized Breast Imaging Reporting and Data System
Correspondence: Zhila Khamnian Email Zhila.khamnian@gmail.com 
(BI-RADS). ${ }^{3}$ BI-RADS was first developed by American College of Radiology (ACR) in 2003 and is used for grading the breast lesions based on the probability of malignancy. ${ }^{4}$ Breast ultrasonography (US) is a complementary technique for further evaluation of suspected mammography lesions. It is the method of choice for the assessment of dense breasts in younger females. ${ }^{5}$ It is relatively inexpensive, safe, and easily applicable without imposing additional radiation or contrast medium. US has shown promising results in differentiation of malignant lesions from benign ones. ${ }^{6}$ A similar BI-RADS classification is also used for grading of the lesions detected by US imaging. ${ }^{7}$

Recent studies have proposed that additional information about the elasticity and vascularity of breast lesions might improve the diagnostic accuracy of conventional B-mode US. ${ }^{8-10}$ Sonoelastography (SE) is a diagnostic technique to determine the relative elasticity of suspected masses in comparison to the surrounding tissue. Malignant masses are stiffer than the normal tissues, so measuring the tissue strain by compressing the sonography transducer provides information regarding the strain of breast lesions in relation to the surrounding fatty tissue (strain ratio) and breast lesion elasticity in SE compared to its length in B mode sonography (E/B ratio). ${ }^{11,12}$ Moreover, color Doppler US helps to explore vascularity within the tumor and provides information regarding the angiogenesis in breast masses. ${ }^{13}$ The current study is conducted to determine the diagnostic yield of conventional US, SE, and color Doppler US and different features of SE, including strain ratio and $\mathrm{E} / \mathrm{B}$ ratio in differentiating malignant breast lesions. We hypothesized that $\mathrm{SE}$ and color Doppler imaging would increase the diagnostic performance of B-mode US.

\section{Materials and Methods}

\section{Ethical Information}

The study was conducted according to the Declaration of Helsinki and the protocol was approved by University/ regional research ethics committee of Tabriz University of Medical Sciences (IR.TBZMED.REC.1397.070). A written informed consent was obtained from all the participants.

Patient information Sheet (PIS) was encoded and anonymized. Patients were not charged for extra procedures.

\section{Study Population}

This prospective study was conducted on women referred to Alzahra university hospital of Tabriz for annual screening of breast cancer between May 2017 and December 2018. Among females who underwent mammography imaging, those with a breast lesion suspected for malignancy with BIRADS $>>3$ were considered and participants with the following criteria were excluded: Age $<18$ years, women with a history or current malignancy, a history of breast surgery within the previous 12 months, pregnancy, and lactation. A sample of the eligible participants was randomly selected to be included in the study. The sample size was calculated, using the breast cancer prevalence and the number of patients presenting to the hospital for annual screening of breast cancer with the power of $80 \%$.

\section{US Examinations and Image Evaluation}

Bilateral whole breast imaging was performed using UGEO WS80A (Samsung Medison, Korea) with a 12$\mathrm{MHz}$ linear transducer by two experienced radiologists (10 years of experience).

First, conventional sonography images were obtained, and target lesions and their echogenicity and size were defined by the first expert radiologist (also 10 years of experience) who was blinded to mammography reports. After the B-mode US was performed, SE and color Doppler images were studied by the second radiologist (blinded to B-mode ultrasonography) using the transducers and were saved as cine clips for further evaluations. In SE and color Doppler assessment, images were displayed on split screens with B-mode findings (Figure 1A). Fivegrade SE scoring (Tsukuba-score) was used based on the comparison of the strain in the lesion with surrounding tissue (Figure 1B). For this purpose, a region of interest (ROI) including the lesion and surrounding tissue was considered and a slight compression was applied. Strain differences were illustrated by colors according to different amounts of tissue movement within the ROI. In this scoring system, lesions with complete hypo-echoic features were scored 1 (entirely green). Lesions with some no strain regions were scored 2 (green and blue mosaic pattern). Lesions with peripheral strain and without central strain were graded 3 (peripherally green and centrally blue). Lesions mostly without strain while with strain in the surrounding area were graded 4 (entirely blue with green background in the surrounding area) and lesions 

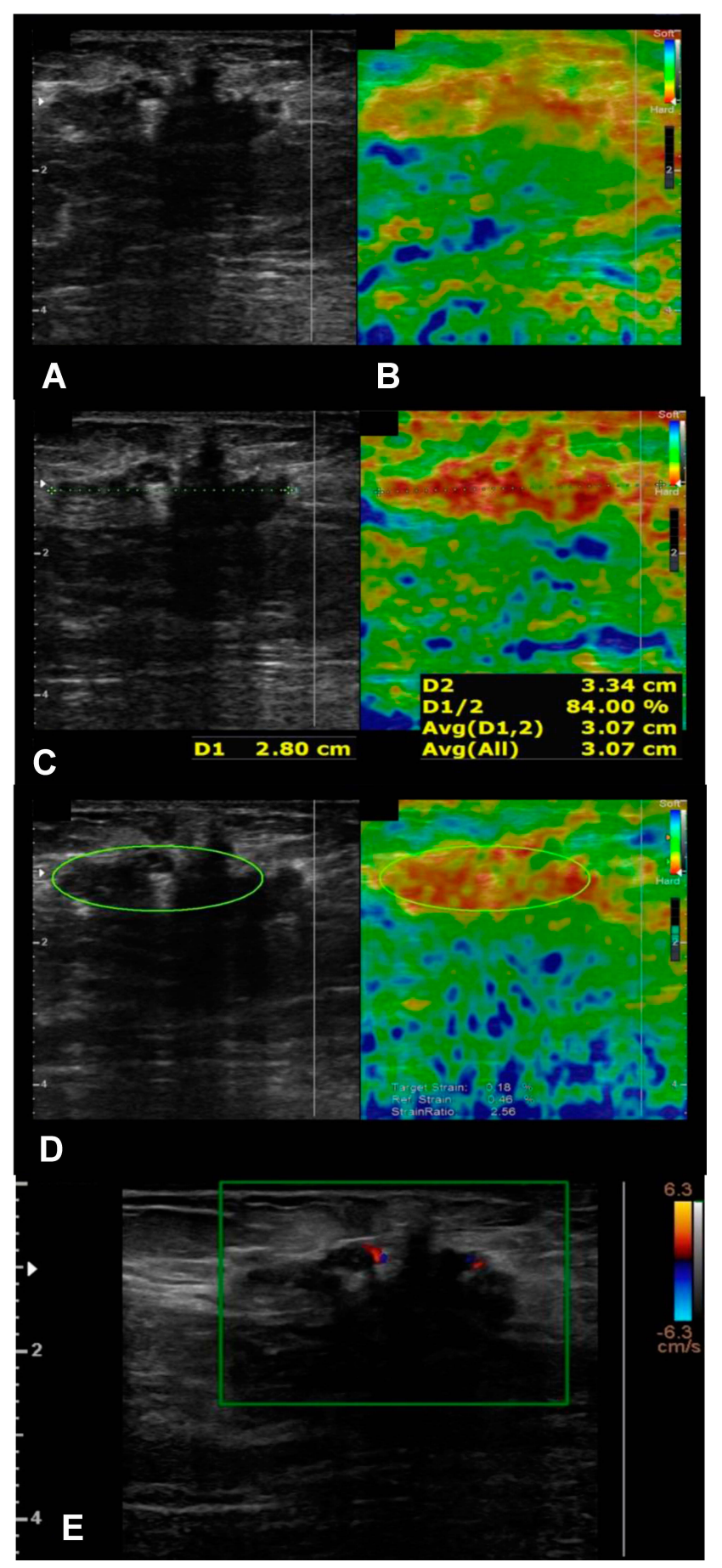

Figure I Sonography images of a 39-year-old woman. (A) B-mode US views showing a hypoechoic breast lesion. (B) Elastography image with orange to red color regions, suggesting of SE score of 4 . (C) Elastography image with side by side B-mode view, the B-mode diameter of the lesion is $2.80 \mathrm{~cm}$ and SE diameter is 3.34 with E/B ratio of I.I9. (D) Elastography image with side by side B-mode view with SR of 2.56. (E) Color Doppler US image with two intralesional vascularity, suggesting a Doppler score of 2 .

without strain in the target area and surrounding tissue were graded 5 (entirely blue). Elasticity to B mode ratio (E/B ratio) was also measured by dividing the length of a lesion on elastography to the length of the lesion on
B-mode US (Figure 1C). Strain ratio (SR) was calculated by the ratio of mean lesion strain to surrounding fatty tissue at the same depth (Figure 1D).

Color Doppler lesion images were also performed by the second radiologist. In this regard, an ROI including mostly the lesion and the least possible amount of surrounding tissue was considered and the lowest pressure was used in order not to eliminate thin vessels. The setting used for optimal color was as follows: low wall filter of $50 \mathrm{~Hz}$, pulse repetition frequency of $700 \mathrm{~Hz}$, and intermediate persistence. Color Doppler images were graded from 0 to 2 . Lesions with no visible vessel were graded as 0 . Lesions with a central vessel or a circumferential vessel were graded as 1 and those with more than two vessels or with at least a penetrating vessel were graded as 2 (Figure 1E). B-Mode US images were categorized according to BIRADS (American College of Radiology 2013) classification. In this classification, lesions with BIRADS category of 4 and 5 were probable of malignancy.

\section{Biopsy Methods}

All core biopsies were performed under US guidance for all lesions, and pathology results were available for all the lesions.

\section{Statistical Analysis}

The sensitivity, specificity, and accuracy values were analyzed for each conventional US (B-mode US), SE, SR, E/B ratio, and color Doppler imaging. Receiver operating curve (ROC) analysis was used to determine the cutoff values. Diagnostic accuracy was measured by the Youden Index (YI). The ROC curves were delineated using medCalc.ink software. Boxplots were drawn by GraphPad.prism v.6.0.7 Ink software. All statistical analyses were performed using the Statistical Package for the Social Sciences (SPSS Version 22 for Windows, IBM, Armonk, NY, USA). In normal distribution analysis, the distribution of SR and $\mathrm{E} / \mathrm{B}$ was normal in benign lesions but not in malignant ones. Then, MannWhitney $U$-test was used to compare means.

To determine the efficacy of SE and color Doppler imaging as complementary tools, we accumulated the overall accuracy of B-Mode + color Doppler, B-Mode + SE score, B-Mode + SE score and its two other features $(\mathrm{SR}+\mathrm{E} / \mathrm{B})$. The accuracy of the cumulative variables was compared to gain the highest diagnostic accuracy. 


\section{Results}

During 17 months of study, 110 breast lesions of 104 women aged $42.05 \pm 10.33$ years (18-73) were included. The primary evaluation by conventional B-mode ultrasound categorized $5(4.5 \%)$ of them in BIRADS 3, 56 $(50.9 \%)$ in BIRADS $4 \mathrm{a}, 17(15.4 \%)$ in BIRADS $4 \mathrm{~b}, 23$ $(20.9 \%)$ in BIRADS 4 c, and $9(8.1 \%)$ in BIRADS 5.

Based on the data acquired from SE, the lesions were categorized as follows: score 1:6 (5.4\%) lesions, score 2:30 (27.2\%) lesions, score 3:33 (30\%) lesions, score 4:25 (22.7\%) lesions, and score 5:16 (14.5\%) lesions. According to these results, 41 of the lesions (37.2\%) were suspected as malignant lesions.

The quantitative values for $\mathrm{E} / \mathrm{B}$ ratio were available in 109 lesions. The mean E/B ratio was $1.04 \pm 0.12$ ranging 0.85-1.70. The SR value was also accessible in 71 patients, which was $1.92 \pm 0.96$ (0.50 to 6.0$)$.

The analysis of the Color Doppler results also suggested 53 lesions (48.2\%) to be malignant. The Color Doppler images revealed 57 (51.8\%) lesions with score 0,29 (26.4) with score 1 and 24 (21.8) with score 2.

\section{Pathology Results}

All the lesions underwent biopsy, and the final diagnosis was made by histopathological evaluation. Of all biopsied lesions, $77(70 \%)$ were benign and $33(30 \%)$ were malignant. The final pathologic diagnosis of the lesions is summarized in Table 1

\section{Diagnostic Performance}

The overall diagnostic performance of each method is shown in Table 2.

Table I The Pathological Results of Breast Lesions

\begin{tabular}{|l|l|l|}
\hline \multicolumn{2}{|l|}{ Pathological Diagnosis } & N \\
\hline Benign & Papilloma & $4(5.1 \%)$ \\
& Ductal hyperplasia & $4(5.1 \%)$ \\
& Granulomatosis mastitis & $8(10.3 \%)$ \\
& Abscess & $3(3.8 \%)$ \\
& Fat necrosis & $2(2.5 \%)$ \\
& Fibroadenoma & $15(19.4 \%)$ \\
& Fibrocystic changes & $41(53.2 \%)$ \\
& Total & 77 \\
\hline \multirow{2}{*}{ Malignant } & Ductal carcinoma & $25(75.7 \%)$ \\
& Mucinous carcinoma & $1(3 \%)$ \\
& Lobular carcinoma & $6(18.1 \%)$ \\
& Papillary carcinoma & $1(3 \%)$ \\
& Total & 33 \\
\hline
\end{tabular}

Table 2 The Number of Benign and Malignant Lesions Diagnosed by B-Mode Ultrasound, Sonography Elastography, Color Doppler US, E/B Ratio, and Strain Ratio According to Pathological Results

\begin{tabular}{|l|l|l|}
\hline \multirow{2}{*}{} & \multicolumn{2}{|l|}{ Pathological Result } \\
\cline { 2 - 3 } & Benign (N= 77) & Malignant (N= 33) \\
\hline B-mode ultrasound & $60 / 77(77.9 \%)$ & $32 / 33(97.0 \%)$ \\
Sonography elastography & $67 / 77(87.0 \%)$ & $31 / 33(93.9 \%)$ \\
Color Doppler US & $51 / 77(66.2 \%)$ & $27 / 33(81.8 \%)$ \\
E/B ratio & $59 / 76(77.6 \%)$ & $24 / 33(72.7 \%)$ \\
Strain ratio & $39 / 49(79.6 \%)$ & $17 / 22(77.3 \%)$ \\
\hline
\end{tabular}

Using receiver operating characteristic (ROC) analysis, B-mode US revealed a diagnostic accuracy of $74.9 \%$. It had a sensitivity of $97.0 \%$ and specificity of $77.9 \%$ with AUC of 0.95 for discriminating malignant lesions from benign ones.

The ROC curves for all different tools are shown in Fig 2. We considered SE score $>3$ as optimal cutoff value for malignancy detection, with a sensitivity of $93.9 \%$ and specificity of $87.0 \%$ with AUC of 0.95 . SE scoring had a diagnostic accuracy of $80.9 \%$.

Color Doppler imaging with a criterion of score $>0$ for malignant lesions revealed a sensitivity of $81.8 \%$ and specificity of $66.2 \%$ with AUC of 0.78 . Color Doppler scoring detected malignant lesions with a diagnostic accuracy of $48.0 \%$.

The mean E/B ratio for benign lesions was $1.03 \pm 0.13$ and for malignant lesions was $1.08 \pm 0.07(\mathrm{p}<0.01)$ (Figure 3A). E/B ratio with cutoff value of $1.05 \mathrm{had}$ a sensitivity of $72.7 \%$, a specificity of $77.6 \%$, AUC of 0.77 , and diagnostic accuracy of $50.3 \%$. The mean SR values for benign and malignant lesions were $1.52 \pm 0.4$ and $2.82 \pm 1.1$, respectively $(\mathrm{p}<0.01)$ (Figure $3 \mathrm{~B})$. SR with cutoff value 1.90 had a sensitivity of $77.3 \%$, a specificity of $79.6 \%$, and a diagnostic accuracy of $56.9 \%$ for detecting malignant lesions (AUC: 0.88 ).

ROC curves demonstrated a significant difference between SE score and Color Doppler US (differences between areas $0.21,95 \%$ confidence interval from 0.09 to $0.34, \mathrm{P}<0.01$ ) for differentiation of malignant breast lesions. There was also a significant difference in ROC curves of SE score and E/B ratio (differences between areas $0.18,95 \%$ confidence interval from 0.07 to 0.29 , $\mathrm{P}<0.01)$ and ROC curves of Color Doppler US and SR (the differences between areas $0.15,95 \%$ confidence interval from 0.03 to $0.026, \mathrm{P}<0.01)$. ROC curves also 


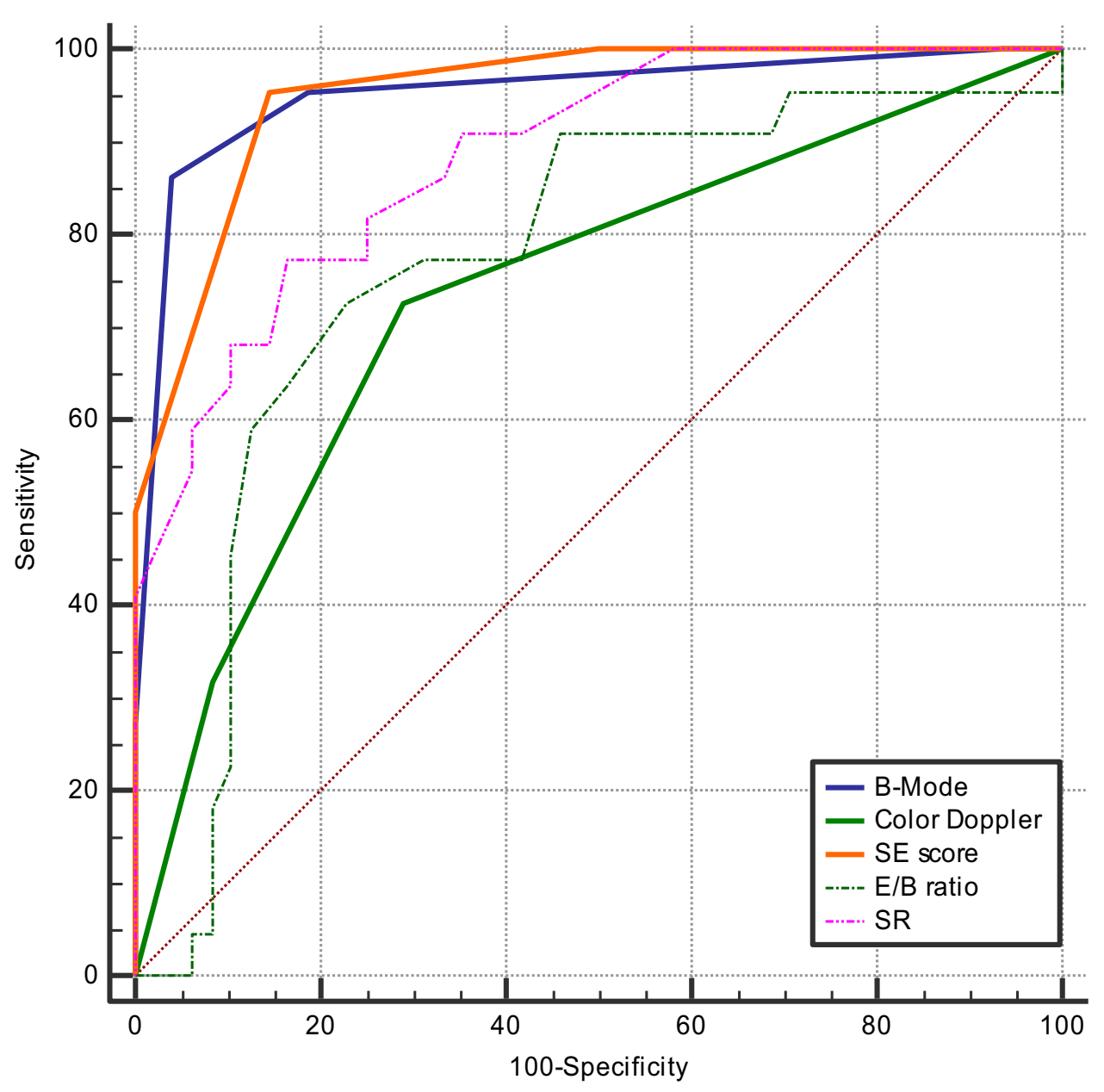

Figure 2 Receiver operating characteristic (ROC) curves for B-mode ultrasound, sonography elastography (SE) score, E/B ratio, strain ratio (SR), and color Doppler.

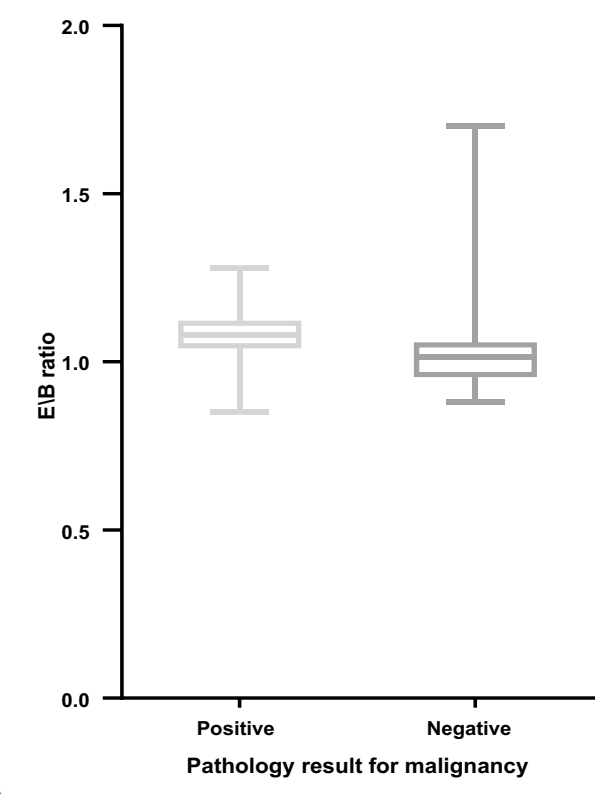

A

Pathology result for malignancy

Figure 3 (A) Boxplot graphs demonstrating a significant difference in elastography E/B ratio for pathology positive and pathology negative breast lesions. Whiskers and Lines delineate $95 \%$ Confidence intervals. (B) Boxplot graphs demonstrating a significant difference in elastography strain ratio for pathology positive and pathology negative breast lesions. Whiskers and Lines delineate $95 \%$ confidence intervals.

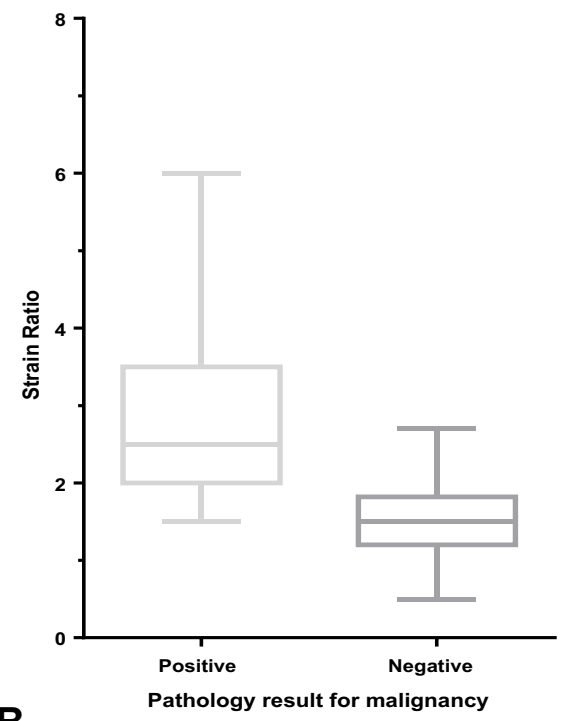

B

Pathology result for malignancy 
demonstrated a significant difference between SR and E/B (the differences between areas $0.11,95 \%$ confidence interval from 0.007 to $0.22, \mathrm{P}=0.03$ ). However, ROC curves did not yield any significant difference between color Doppler scores and E/B ratio (the differences between areas $0.03,95 \%$ confidence interval from -0.13 to 0.20 , $\mathrm{P}=0.68$ ), and ROC curves of SE score and SR (differences between areas $0.06,95 \%$ confidence interval from 0.00 to $0.14, \mathrm{P}=0.07$ ) were not significantly different as well.

\section{Combination of Different Scores}

We used a cumulative variable for each SE score and its featured scales (SR and E/B) + B-mode US as well as Color Doppler + B-mode US. We graded the lesions with SE scores higher than its cutoff $(>3)+1$ and those with scores lower than cutoff -1 . We applied a similar grading approach for SR and E/B scores. The lesions with color Doppler score of 0 were graded -1 and those with Doppler scores of 1 and 2 were graded 0 and +1 , respectively. The sensitivity and specificity for B-Mode + SE score were 93.9\% and $93.5 \%$ (Figure 4) vs $84.8 \%$ and $94.8 \%$ for B-Mode + color Doppler, respectively (Figure 5). The cumulative B-mode $+\mathrm{SE}$ score $+\mathrm{SR}+\mathrm{E} / \mathrm{B}$ resulted in a sensitivity of $93.7 \%$ and specificity of $86.3 \%$ and an accuracy of $80 \%$ (Fig 6). Thus, B-mode + SE score had the highest diagnostic accuracy than other cumulative scores.

\section{Discussion}

\section{Principal Findings}

Breast cancer has a high mortality rate due to the higher stages of malignancy at the time of diagnosis. Hence, more accurate modalities for better diagnosis are required to be used in the primary stages. ${ }^{14}$ In the present study, we used SE and Color Doppler imaging additionally to conventional B-mode ultrasound imaging in women suspected of breast cancers. Three different features of SE were

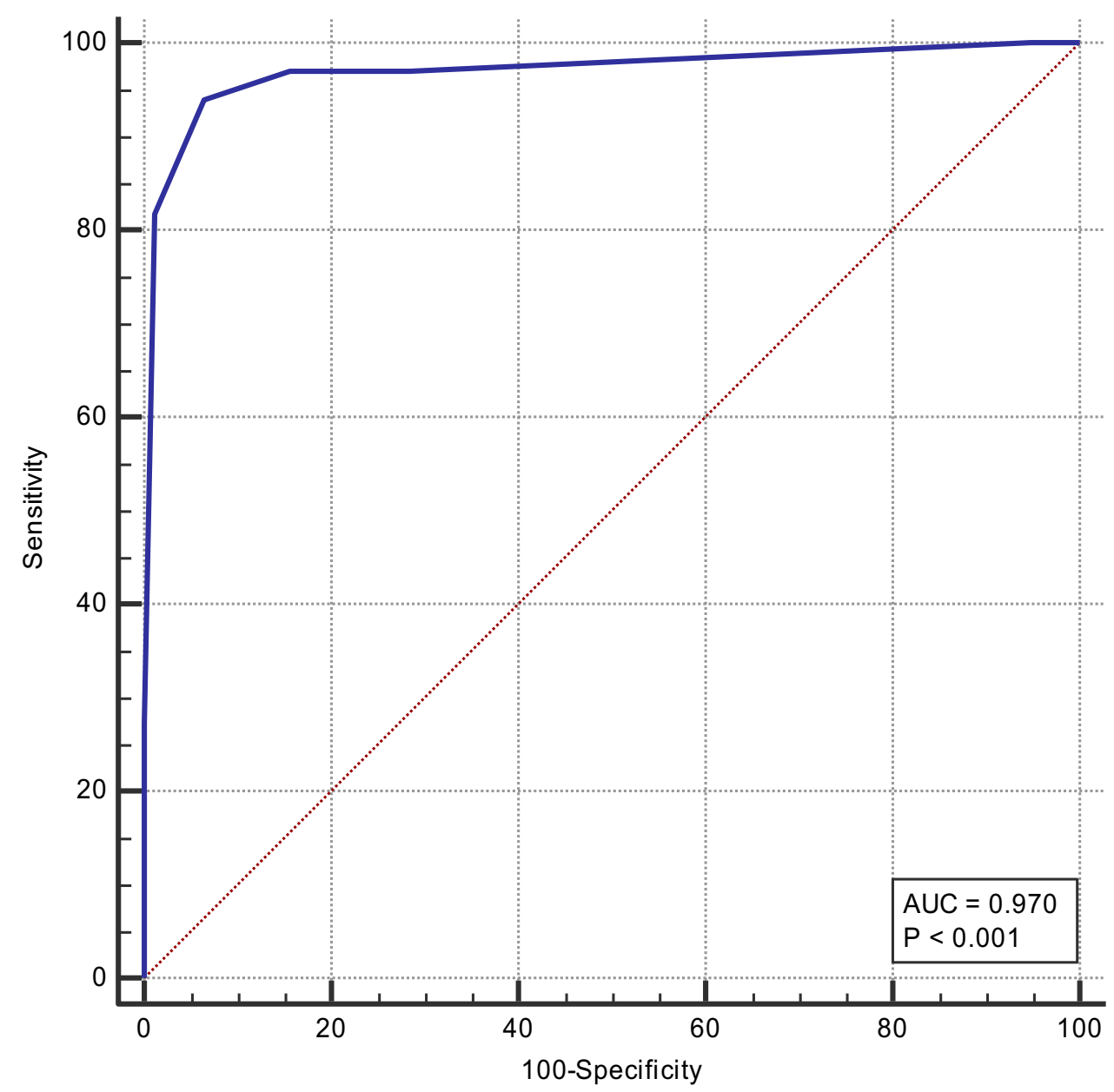

Figure 4 Receiver operating characteristic (ROC) curves for the combination of conventional B-mode ultrasound+ SE score. 


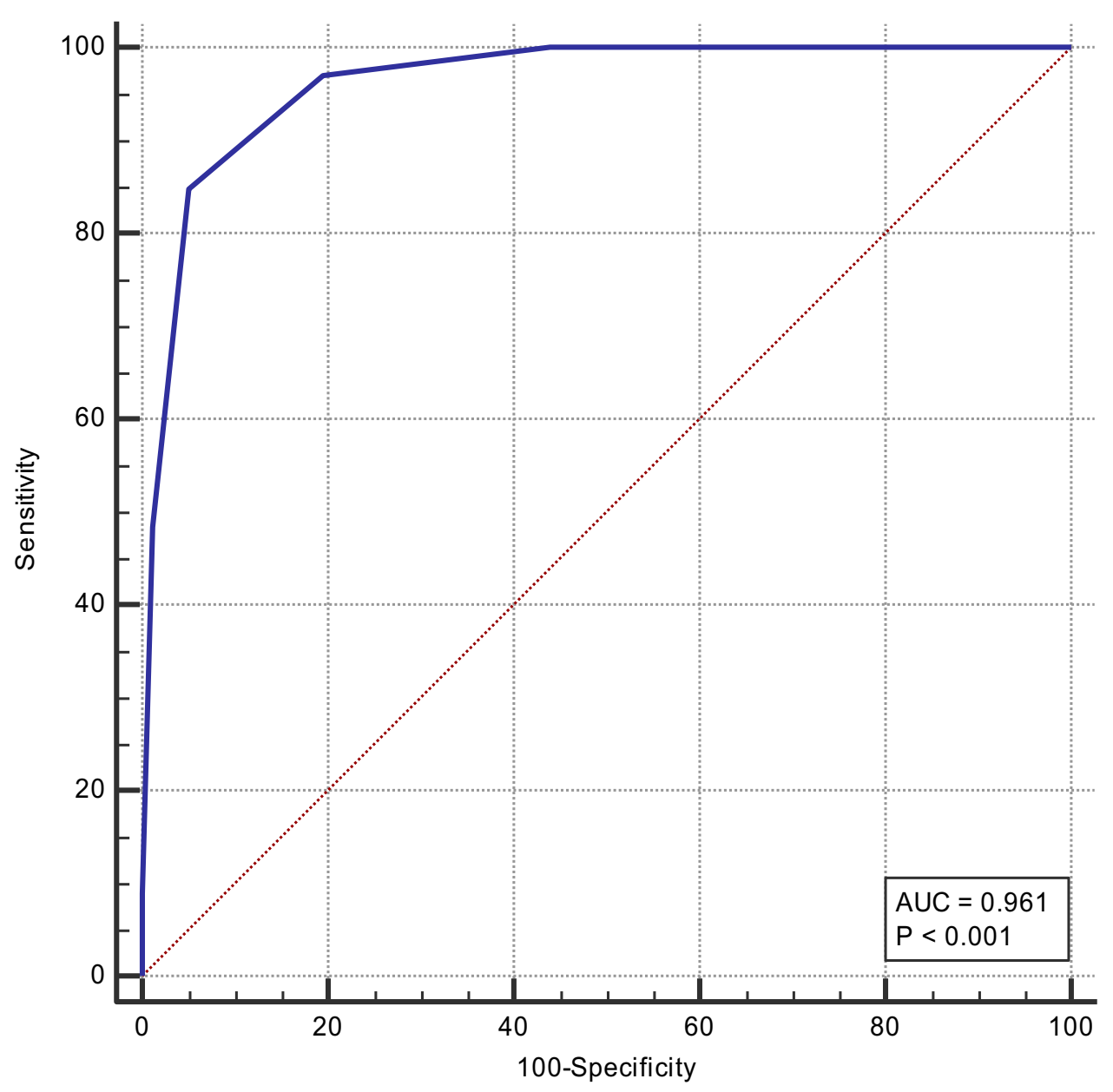

Figure 5 Receiver operating characteristic (ROC) curves for the combination of conventional B-mode ultrasound+ color Doppler.

included in our results: SE score, E/B ratio, and SR. Among 77 benign lesions, SE scoring correctly diagnosed 67 lesions compared to 60 lesions detected by conventional B-mode ultrasound. In terms of malignancy, SE had a similar detection rate to conventional ultrasound. We also found an E/B cutoff value of 1.05 and SR cutoff value of 1.90 for discriminating malignant breast lesions from benign lesions. Among the three different SE features, SE five-point scoring system had a higher diagnostic accuracy than E/B and SR values $(80.9 \%$ vs $50.3 \%$ and $56.9 \%$, respectively). Moreover, cumulative SE score with B-mode results increased the diagnostic accuracy from $74.9 \%$ to $87.4 \%$. On the other hand, conventional B-mode ultrasound was superior to Color Doppler imaging either in benign lesions or in malignancies. Color Doppler scoring could distinguish 51 out of 77 benign lesions and 27 out of 33 malignant lesions, which had a lower diagnostic accuracy than B-mode ultrasound $(48.0 \%$ vs $74.9 \%$ ). In addition, the combination of Color Doppler imaging with B-mode imaging did not improve the diagnostic performance of B-mode ultrasound.

\section{Findings in Relation to Other Studies and Possible Mechanisms for the Findings}

We found that combined use of B-mode ultrasonography with SE score is more effective in differentiating malignant breast lesions than combination of sonography with color Doppler or sonography alone. Similar findings have been reported in previous studies. ${ }^{15-18}$ Hao et al found an increased AUC of B-mode US from 0.73 to 0.87 after the addition of SE. ${ }^{18}$ In the study of Yeo et al, the AUC for combined SE with sonography was 0.86 , which was higher than the sonography with color Doppler $(0.57)$ or sonography alone (0.65). ${ }^{17}$ In our study, the AUC for conventional sonography was higher than the previous studies. The AUC for combined sonography with SE was 0.96, with a slight elevation compared to sonography alone (0.95) and was in a similar range to the cumulative 


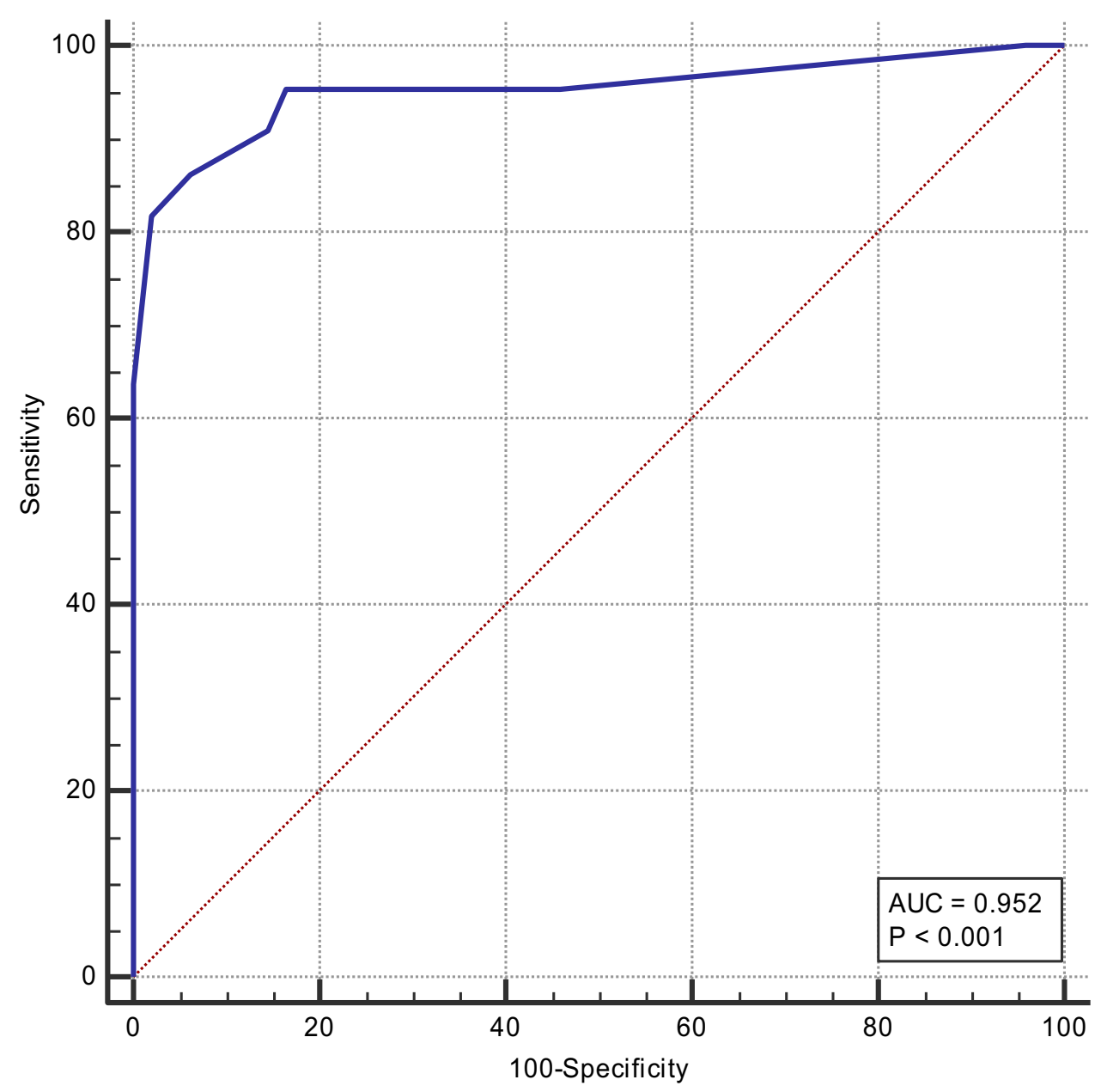

Figure 6 Receiver operating characteristic (ROC) curves for the combination of conventional B-mode ultrasound+ SE score + E/B ratio + SR.

sonography and color Doppler results (0.96). On the other hand, color Doppler imaging had inferior diagnostic results to conventional sonography. Furthermore, the cumulative results of color Doppler with conventional method did not yield a significant improvement in the diagnostic performance of sonography alone. Regardless of the low diagnostic efficacy of color Doppler in our study, there are various reports emphasizing the value of color Doppler imaging in reducing negative biopsies. ${ }^{19-22}$ Elkharbotly et al showed that adding color Doppler imaging would enhance the diagnostic performance of sonography by decreasing the number of false-positive results from 5 to 4 and the number of false-negative results from 10 to 9 . It improved the AUC of sonography from 0.74 to $0.78 .^{22}$ Svensson et al also found that application of vascular morphology in color Doppler imaging elevated the sensitivity of sonography from $97 \%$ to $99 \%$. $^{20}$

In our study, five-point scoring system of $\mathrm{SE}$ was superior to $\mathrm{SR}$ and $\mathrm{E} / \mathrm{B}$ ratio. On the contrary, previous studies have represented a similar or higher diagnostic performance of SR compared with SE and conventional sonography. ${ }^{23-25}$ In the current study, the sensitivity and specificity of E/B ratio were $72.7 \%$, and $77.6 \%$, respectively, and sensitivity and specificity of the SR ratio were $77.3 \%$ and $79.6 \%$, respectively. The diagnostic value of both parameters was in a similar range to other studies. However, both the parameters had inferior diagnostic performance compared to the B-mode US and five-point scoring system of SE. Therefore, the diagnostic performance of SE and B-mode US was higher than the similar studies in this field.

In the present study, the mean SR values for benign and malignant lesions were $1.52 \pm 0.4$ and $2.82 \pm 1.1$, respectively, and 1.9 as the cutoff value had the highest sensitivity and specificity for malignancy detection. The mean SR of malignant lesions and cutoff value were lower than the similar studies. ${ }^{12,26,27}$ The SR values for malignant lesions were 4.9 in the study of Bojanic et al, ${ }^{26} 3.02 \pm 1.3$ in Lee et al, ${ }^{12}$ and 7.9 
\pm 5.8 in Mu et al. ${ }^{27}$ Different SR values may be due to the use of different ultrasonography instruments. We applied UGEO WS80A (Samsung Medison, Korea) in our study, while other imaging instruments in the literature detected higher SR values, such as Logiq Expert 7 ultrasound scanner (General Electric, Fairfield, CT, USA) in the study of Bojanic et al. ${ }^{26}$ EUB-8500 (Hitachi Medical, Tokyo, Japan) in the study of Lee et $\mathrm{al}^{12}$ and $\mathrm{Mu}$ et $\mathrm{al}^{27}$ and Philips iU22 (Philips Healthcare, Bothell, WA, USA) in the study of Alhabshi et al. $^{28}$

\section{Strength and Weakness of the Study}

In this study, we determined the diagnostic values of three different ultrasound modalities and compared two quantitative and a qualitative elastography tools with qualitative Color Doppler results and conventional B-mode ultrasound imaging. To our knowledge, this study is the first in the literature to compare these five diagnostic tools in breast lesion. Our findings improve our understanding of different non-invasive imaging tools and help to increase the diagnostic value of conventional B-mode ultrasonography. We also found new cutoff values for $\mathrm{E} / \mathrm{B}$ ratio and $\mathrm{SR}$ which could be used in further studies to distinguish malignant breast lesions from benign masses. The study was exploratory in nature with a medium sample size. The results were repeated once by the same radiologist without a second radiologist opinion and the interobserver agreement was not obtained. Hence, further studies with greater sample size and contribution of at least two radiologists are required to make a certain conclusion of our cutoff values and imaging results.

\section{Conclusion}

According to the conclusion, differentiation between malignant and benign breast lesions was more precise while using SE than color Doppler US. Also, from a clinical point of view, the combination of SE with B-mode ultrasonography appears to be more useful than using sonography in combination with color Doppler or alone. On the other hand, as concluded, SE five-point scoring system was more accurate in diagnosing malignant lesions.

\section{Acknowledgments}

This project is funded by Tabriz University of Medical Sciences as part of Dr. Moradi's dissertation project for radiology specialty degree. We extend our appreciation to Zahra Seifar and Yousef Asgharzadeh for their technical assistance. We also would like to thank all the participants for their contribution to this research project.

\section{Disclosure}

The authors report no conflicts of interest in this work.

\section{References}

1. Mousavi SM, Montazeri A, Mohagheghi MA, et al. Breast cancer in Iran: an epidemiological review. Breast J. 2007;13(4):383-391. doi:10.1111/j.1524-4741.2007.00446.x

2. Afsharfard A, Mozaffar M, Orang E, Tahmasbpour E. Trends in epidemiology, clinical and histopathological characteristics of breast cancer in Iran: results of a 17 year study. Asian Pac J Cancer Prev. 2013;14(11):6905-6911. doi:10.7314/APJCP.2013.14.11.6905

3. Heidinger O, Heidrich J, Batzler WU, et al. Digital mammography screening in Germany: impact of age and histological subtype on program sensitivity. Breast. 2015;24(3):191-196. doi:10.1016/j. breast.2014.12.009

4. American College of Radiology. Breast Imaging Reporting and Data System. Reston, VA: American College of Radiology; 2003.

5. Athanasiou A, Tardivon A, Ollivier L, Thibault F, El Khoury C, Neuenschwander S. How to optimize breast ultrasound. Eur J Radiol. 2009;69(1):6-13. doi:10.1016/j.ejrad.2008.07.034

6. Meel-van den Abeelen ASS, Weijers G, van Zelst JCM, Thijssen JM, Mann RM, de Korte CL. 3D quantitative breast ultrasound analysis for differentiating fibroadenomas and carcinomas smaller than $1 \mathrm{~cm}$. Eur J Radiol. 2017;88:141-147. doi:10.1016/j.ejrad.2017.01.006

7. Li X-L, Xu H-X, Bo X-W, et al. Value of virtual touch tissue imaging quantification for evaluation of ultrasound breast imaging-reporting and data system category 4 lesions. Ultrasound Med Biol. 2016;42 (9):2050-2057. doi:10.1016/j.ultrasmedbio.2016.04.002

8. Li -D-D, Xu H-X, Guo L-H, et al. Combination of two-dimensional shear wave elastography with ultrasound breast imaging reporting and data system in the diagnosis of breast lesions: a new method to increase the diagnostic performance. Eur Radiol. 2016;26 (9):3290-3300. doi:10.1007/s00330-015-4163-8

9. Kim S-Y, Park JS, Koo HR. Combined use of ultrasound elastography and B-mode sonography for differentiation of benign and malignant circumscribed breast masses. J Ultrasound Med. 2015;34 (11):1951-1959. doi:10.7863/ultra.14.11027

10. Arslan S, Uslu N, Ozturk FU, Akcay EY, Tezcaner T, Agildere AM. Can strain elastography combined with ultrasound breast imaging reporting and data system be a more effective method in the differentiation of benign and malignant breast lesions? J Med Ultrason. 2017;44(4):289-296. doi:10.1007/s10396-017-0772-y

11. Gheonea IA, Stoica Z, Bondari S. Differential diagnosis of breast lesions using ultrasound elastography. Indian $J$ Radiol Imaging. 2011;21(4):301-305. doi:10.4103/0971-3026.90697

12. Lee JH, Kim SH, Kang BJ, et al. Role and clinical usefulness of elastography in small breast masses. Acad Radiol. 2011;18(1):74-80. doi:10.1016/j.acra.2010.07.014

13. Lee S-W, Choi HY, Baek SY, Lim SM. Role of color and power doppler imaging in differentiating between malignant and benign solid breast masses. J Clin Ultrasound. 2002;30(8):459-464. doi: $10.1002 /$ jcu. 10100

14. Hashemzadeh S, Mehrafsa B, Kakaei F, et al. Diagnostic accuracy of a 64-slice multi-detector CT scan in the preoperative evaluation of periampullary neoplasms. J Clin Med. 2018;7:5. doi:10.3390/ jem7050091

15. Li L, Zhou X, Zhao X, et al. B-mode ultrasound combined with color doppler and strain elastography in the diagnosis of non-mass breast lesions: a prospective study. Ultrasound Med Biol. 2017;43 (11):2582-2590. doi:10.1016/j.ultrasmedbio.2017.07.014

16. Cho N, Jang M, Lyou CY, Park JS, Choi HY, Moon WK. Distinguishing benign from malignant masses at breast US: combined US elastography and color doppler US-influence on radiologist accuracy. Radiology. 2012;262(1):80-90. doi:10.1148/radiol.11110886 
17. Yeo SH, Kim GR, Lee SH, Moon WK. Comparison of ultrasound elastography and color doppler ultrasonography for distinguishing small triple-negative breast cancer from fibroadenoma. J Ultrasound Med. 2018;37(9):2135-2146. doi:10.1002/jum.14564

18. Hao SY, Jiang QC, Zhong WJ, et al. Ultrasound elastography combined with BI-RADS-US classification system: is it helpful for the diagnostic performance of conventional ultrasonography? Clin Breast Cancer. 2016;16(3):e33-e41. doi:10.1016/j.clbc.2015. 10.003

19. Cosgrove DO, Kedar RP, Bamber JC, et al. Breast diseases: color Doppler US in differential diagnosis. Radiology. 1993;189 (1):99-104. doi:10.1148/radiology.189.1.8372225

20. Svensson WE, Pandian AJ, Hashimoto H. The use of breast ultrasound color Doppler vascular pattern morphology improves diagnostic sensitivity with minimal change in specificity. Ultraschall Med. 2010;31(5):466-474. doi:10.1055/s-0028-1109478

21. Kwak JY, Kim E-K, Kim MJ, Choi SH, Son E, Oh KK. Power Doppler sonography: evaluation of solid breast lesions and correlation with lymph node metastasis. Clin Imaging. 2008;32(3):167-171. doi:10.1016/j.clinimag.2007.12.004

22. Elkharbotly A, Farouk HM. Ultrasound elastography improves differentiation between benign and malignant breast lumps using B-mode ultrasound and color Doppler. Egypt J Radiol Nucl Med. 2015;46(4):1231-1239. doi:10.1016/j.ejrnm.2015.06.005
23. Parajuly SS, Lan PY, Yun MB, Gang YZ, Hua Z. Diagnostic potential of strain ratio measurement and a 5 point scoring method for detection of breast cancer: chinese experience. Asian Pac J Cancer Prev. 2012;13(4):1447-1452. doi:10.7314/APJCP.2012.13.4.1447

24. Cantisani V, Maceroni P, D'Andrea V, et al. Strain ratio ultrasound elastography increases the accuracy of colour-Doppler ultrasound in the evaluation of Thy-3 nodules. a bi-centre university experience. Eur Radiol. 2016;26(5):1441-1449. doi:10.1007/s00330-015-3956-0

25. Thomas A, Degenhardt F, Farrokh A, Wojcinski S, Slowinski T, Fischer T. Significant differentiation of focal breast lesions: calculation of strain ratio in breast sonoelastography. Acad Radiol. 2010;17 (5):558-563. doi:10.1016/j.acra.2009.12.006

26. Bojanic K, Katavic N, Smolic M, et al. Implementation of elastography score and strain ratio in combination with B-mode ultrasound avoids unnecessary biopsies of breast lesions. Ultrasound Med Biol. 2017:43(4):804-816. doi:10.1016/j.ultrasmedbio.2016.11.019

27. Mu W-J, Zhong W-J, Yao J-Y, et al. Ultrasonic elastography research based on a multicenter study: adding strain ratio after 5-point scoring evaluation or not. PLoS One. 2016;11(2):e0148330. doi:10.1371/ journal.pone. 0148330

28. Alhabshi SMI, Rahmat K, Abdul Halim N, et al. Semi-quantitative and qualitative assessment of breast ultrasound elastography in differentiating between malignant and benign lesions. Ultrasound Med Biol. 2013;39(4):568-578. doi:10.1016/j.ultrasmedbio.2012.10.016
International Journal of General Medicine

\section{Publish your work in this journal}

The International Journal of General Medicine is an international, peer-reviewed open-access journal that focuses on general and internal medicine, pathogenesis, epidemiology, diagnosis, monitoring and treatment protocols. The journal is characterized by the rapid reporting of reviews, original research and clinical studies

\section{Dovepress}

across all disease areas. The manuscript management system is completely online and includes a very quick and fair peer-review system, which is all easy to use. Visit http://www.dovepress.com/ testimonials.php to read real quotes from published authors. 